\title{
TECNOLOGÍAS MÓVILES PARA LA SALUD PÚBLICA EN EL PERÚ: LECCIONES APRENDIDAS
}

\author{
Eloy F. Ruiz',a, Álvaro Proaño ${ }^{1, a}$, Oscar J. Ponce ${ }^{1, a,{ }^{*}}$, Walter H. Curioso ${ }^{2,3, b}$ \\ RESUMEN \\ En los últimos años, las tecnologías de la comunicación móvil se han expandido de manera rápida tanto en los países \\ desarrollados como en vías de desarrollo. Estos países, incluyendo a Perú, son escenarios ideales donde las herramientas \\ de la salud móvil (mSalud) se convierten en excelentes oportunidades para brindar y hacer más inclusivo los servicios de \\ salud. La literatura revisada muestra que las intervenciones de mSalud en nuestro país, presentan un enorme potencial \\ para mejorar el acceso y calidad de la los servicios de salud, aumentando la efectividad de los programas de salud pública y \\ reduciendo los costos de la asistencia sanitaria. No obstante, se requiere de mayor investigación en estas áreas para com- \\ prender en su totalidad el potencial de la mSalud para que puedan ser base y sustento de políticas de estado innovadoras, \\ integradas al sistema de salud y costo-efectivas para la mejora en la atención e inclusión en salud en Perú.
}

Palabras clave: Proyectos de tecnologías de información y comunicación; Teléfono celular; Mensaje de texto; Salud Pública (fuente: DeCS BIREME).

\section{MOBILE HEALTH FOR PUBLIC HEALTH IN PERU: LESSONS LEARNED}

\begin{abstract}
Mobile communication technologies have become more prevalent in developed and developing countries. These countries -including Peru- are becoming an ideal setting where mobile health (mHealth) projects can provide better health services. The reviewed literature shows that the mHealth interventions have enormous potential to improve access and the quality of health services, increasing the effectiveness of public health programs and reducing healthcare costs. It is noticeable that these projects have a positive impact; however, despite the current information, more research is needed to understand $\mathrm{mHealth}$ in-depth. These projects are the foundation for future health policies that will help the Peruvian health system to be more inclusive and more effective.
\end{abstract}

Key words: Information technologies and communication projects; Cellular phone; Text messaging; Public health (source: MeSH NLM).

\section{INTRODUCCIÓN}

En los últimos años, las tecnologías de la comunicación móvil se han expandido de manera rápida tanto en los países desarrollados como en vías de desarrollo ${ }^{(1,2)}$. De acuerdo con la Unión Internacional de Telecomunicaciones, la cantidad de usuarios de teléfonos celulares en el mundo se aproximó a 7 billones a finales del $2014{ }^{(3)}$. En los países en vías de desarrollo, la gran accesibilidad y disponibilidad de los celulares ha avanzado más rápido que el desarrollo de otras infraestructuras de comunicación ${ }^{(2,4)}$, y en consecuencia, más personas tendrían acceso a teléfonos móviles que a los servicios de agua y saneamiento ${ }^{(5)}$. La popularidad de las tecnologías móviles se ha visto reflejada con una penetrancia del $90 \%$ en el mundo en desarrollo ${ }^{(3)}$ por lo que la relación íntima que se ha generado entre el usuario y su celular podría ser una pieza clave para para acercarse, interactuar y brindar servicios a las personas ${ }^{(2,4,6)}$.

Debido a que los servicios de salud en los países en vías en desarrollo tienen limitaciones y no son igualmente accesibles para las personas que viven en zonas urbanas y rurales ${ }^{(7)}$, las tecnologías móviles surgen como una opción innovadora para la asistencia sanitaria ${ }^{(8)}$. Estos países, incluyendo a Perú, son escenarios ideales

\footnotetext{
Facultad de Medicina. Universidad Peruana Cayetano Heredia. Lima, Perú.

Dirección de Evaluación y Gestión del Conocimiento. Consejo Nacional de Ciencia, Tecnología e Innovación Tecnológica (CONCYTEC). Lima, Perú.

Department of Biomedical Informatics and Medical Education. School of Medicine, University of Washington. Seattle, Washington, EE. UU.

Estudiante de Medicina; b médico, maestro en Salud Pública, doctor en Informática Biomédica.

Recibido: : 03-04-15 Aprobado: 27-05-15
}

Citar como: Ruiz EF, Proaño A, Ponce OJ, Curioso WH. Tecnologías móviles para la salud pública en el Perú: lecciones aprendidas. Rev Peru Med Exp Salud Publica. 2015;32(2):364-72. 
donde las herramientas de la salud móvil se convierten en excelentes oportunidades para brindar y hacer más inclusivo los servicios de salud ${ }^{(1,2,6,7)}$.

\section{¿QUÉ ES LA SALUD MOVIL?}

La salud móvil o mSalud se define como la aplicación de telecomunicaciones móviles y otras tecnologías inalámbricas para transmitir y brindar asistencia e información médica a través de dispositivos electrónicos móviles o MEDs (mobile electronic devices) como los celulares, asistentes digitales personales, teléfonos PDA (BlackBerry), teléfonos inteligentes o smartphones (ej. iPhone), asistentes digitales empresariales (enterprise digital assistant), reproductores de audio digital (ej. reproductores MP3, MP4, iPod), videoconsolas portátiles (ej. PlayStation Portable, Nintendo DS), Tablets (ej. iPad) y smartbooks ${ }^{(1,4,9)}$.

Las tecnologías móviles presentan ventajas sobre otras TIC (tecnologías de información y comunicación) dentro del marco de la salud: los dispositivos electrónicos móviles son económicos, inalámbricos, portátiles, soportan aplicaciones de software, proporcionan comunicación continua desde cualquier lugar (incluyendo, pero no limitándose a mensajes de texto, llamadas y conectividad a Internet) y sobre todo, logran empoderar al usuario, por lo que se convierten en herramientas importantes para los objetivos de la salud móvil ${ }^{(1,4,8,9)}$.

Muchos países del mundo están utilizando las tecnologías móviles para transformar sus sistemas de salud ${ }^{8,9)}$. Gran parte de la evidencia de la efectividad de la mSalud proviene de los trabajos realizados en los países industrializados ${ }^{(1)}$, sin embargo, con la expansión de la cobertura de la telefonía móvil en el mundo en desarroIlo ${ }^{(3)}$, y la necesidad de proporcionar atención en salud en los mismos, la mSalud y los dispositivos electrónicos móviles se están convirtiendo en un medio para mejorar: 1) el acceso a la información, promoción y educación en salud; 2) el diagnóstico de enfermedades; 3) el apoyo, control, seguimiento y adherencia al tratamiento médico; 4) la recolección de información, monitoreo de enfermedades y vigilancia epidemiológica; 5) el entrenamiento al personal de salud; entre otros $(1,2,4,6,9,10)$.

\section{SITUACIÓN EN EL PERÚ}

En el año 2009, las Naciones Unidas publicó uno de los primeros reportes a nivel mundial (1) que incluyó nueve proyectos relacionados a mSalud de Latinoamérica (LA), incluyendo cuatro de Perú, enfocándose principalmente en la adherencia al tratamiento, soporte al diagnóstico, la recolección de información y el entrenamiento al personal de salud (Tabla 1). Este informe discutió el estado actual de las investigaciones en mSalud en LA con la finalidad de impulsar más iniciativas con estas tecnologías móviles, y destacó al Perú como uno de los países con más iniciativas de mSalud en países en vías de desarrollo. Asimismo, el reporte menciona que los programas en mSalud generan impactos demostrables y medibles, principalmente en la eficiencia de la asistencia sanitaria ${ }^{(1)}$. Por otro lado, a pesar que en los últimos años ha aumentado el interés en la aplicación y maximización de los beneficios de estas tecnologías en países en vías de desarrollo, todavía no hay claridad sobre el estado y la dirección de la investigación en mSalud, sobre todo en nuestro país. Por este motivo, el objetivo de este trabajo es revisar y discutir las lecciones aprendidas de los proyectos relacionados a mSalud en el Perú.

Para cumplir el objetivo, tres de los autores (EFR, AP y OJP) realizaron la búsqueda bibliográfica de artículos sobre el uso de aplicaciones de mSalud en el Perú. Se generó una estrategia de búsqueda para MEDLINE/ PubMed (utilizando términos MeSH): [Peru] AND ((short message service OR mensaje de texto) OR (phones) OR (telefono OR celular) OR (mobile health OR salud movil) OR (m-health OR mhealth) OR (PDA OR Personal digital assistants) OR (smartphone)). En caso de discordancia, se consultó con un cuarto autor (WHC) y se llegó a un consenso. Esta estrategia se modificó para realizar la búsqueda las bases de datos SciELO, RedALyC, LILACS y LIPECS.

Se encontraron un total de 246 publicaciones de los cuales se seleccionaron $19^{(11-29)}$ luego de excluir a: 1) artículos que no estaban relacionados al uso de mSalud en Perú y 2) revisiones temáticas. La Figura 1 muestra el diagrama de flujo de la selección de los artículos, así como los motivos

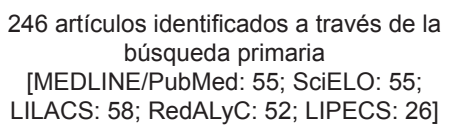

246 artículos identificados a través de la búsqueda primaria

[MEDLINE/PubMed: 55; SciELO: 55;

LILACS: 58; RedALyC: 52; LIPECS: 26]

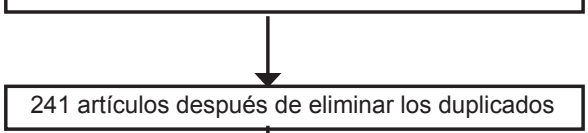

217 artículos excluidos por no estar relacionados al uso de mSalud en Perú

24 artículos evaluados a texto completo

5 artículos excluidos por tratarse de revisiones sistemáticas

24 artículos evaluados a texto completo

Figura 1. Flujo de selección de artículos 
Tabla 1. Proyectos de mSalud destacados en América Latina según Naciones Unidas ( ${ }^{1}$ )

Proyecto Tema Descripción

\begin{tabular}{|c|c|c|}
\hline Argentina & & \\
\hline $\begin{array}{l}\text { Digital Inclusion Kit } \\
\text { in Health and Higher } \\
\text { Education }\end{array}$ & $\begin{array}{l}\text { Sistemas de apoyo a } \\
\text { la toma de decisiones }\end{array}$ & $\begin{array}{l}\text { El kit permite a los teléfonos inteligentes conectarse de forma inalámbrica } \\
\text { a las herramientas de diagnóstico (como electrocardiogramas, rayos X, } \\
\text { tomografías), para realizar diagnósticos sofisticados a distancia. }\end{array}$ \\
\hline
\end{tabular}

Brasil Virtual Health Pet $\quad \begin{aligned} & \text { Adherencia al } \\ & \text { tratamiento }\end{aligned}$
Se ha utilizado la popularidad de las mascotas virtuales para mejorar la adherencia al tratamiento médico de los pacientes en Brasil. La mascota virtual interactúa con el paciente para recordarles que deben tomar sus medicamentos a tiempo y controlar su salud en general.

$\begin{array}{lll}\text { Nokia Data Gathering } & \begin{array}{l}\text { Recolección de } \\ \text { información y vigilancia } \\ \text { epidemiológica }\end{array} & \begin{array}{l}\text { El sistema permite la recopilación de los datos de la propagación del virus } \\ \text { una conexión inalámbrica para su análisis inmediato, con lo que el tiempo } \\ \text { empleado en la recolección de datos se redujo significativamente. }\end{array}\end{array}$

\section{Caribe}

Enhancing Nurses

Access for Care Quality

and Knowledge through Technology
Sistemas de apoyo a la toma de decisiones
Este sistema empodera a las enfermeras mediante capacitación y acceso a información a través de PDA, en particular en las áreas de medicamentos y apoyo al tratamiento.

\section{Guatemala} Primary Healthcare Nursing Telemedicina
Promotion Program
Uno de los componentes de este programa es un curso de enfermería virtual que se imparte a través de teléfonos móviles que se utilizan para vincular sus comunidades rurales con especialistas de salud en las zonas urbanas.

\section{Perú}

\begin{tabular}{|c|c|c|}
\hline Nacer & Telemedicina & $\begin{array}{l}\text { Nacer permite compartir datos acerca de la salud materna e infantil en } \\
\text { zonas remotas con otros médicos más especializados a través de cualquier } \\
\text { teléfono celular con el objetivo de solucionar problemas de diagnóstico y } \\
\text { monitoreo de pacientes. }\end{array}$ \\
\hline
\end{tabular}

$\begin{array}{lll}\text { Colecta-PALM } & \begin{array}{l}\text { Adherencia al } \\ \text { tratamiento }\end{array} & \begin{array}{l}\text { Los resultados sugieren que los PDA pueden ser una manera apropiada } \\ \text { para apoyar la adherencia antirretroviral y el sexo más seguro para } \\ \text { personas con VIH. }\end{array} \\ \text { Cell-PREVEN } & \begin{array}{l}\text { Recolección de } \\ \text { información y vigilancia } \\ \text { epidemiológica }\end{array} & \begin{array}{l}\text { El sistema mostró que los teléfonos celulares son un medio viable de } \\ \text { recoleción y transmisión de datos en tiempo real en comunidades } \\ \text { remora crear un sofisticado sistema de vigilancia epidemiológica en } \\ \text { tiempo real aplicado en salud pública. }\end{array}\end{array}$

\begin{tabular}{|c|c|c|}
\hline ISAMAR & $\begin{array}{l}\text { Recolección de } \\
\text { información y vigilancia } \\
\text { epidemiológica }\end{array}$ & $\begin{array}{l}\text { Es un sistema de vigilancia de enfermedades, que permite a los usuarios } \\
\text { transmitir o acceder a los datos a través de teléfonos móviles. Las alertas } \\
\text { de brotes de enfermedades se envían a través de múltiples mecanismos } \\
\text { (mensajes de texto, correo de voz y correo electrónico) en tiempo real, } \\
\text { facilitando el reporte de enfermedades. }\end{array}$ \\
\hline
\end{tabular}

PDA: personal digital assistant

de exclusión. Se agruparon las iniciativas de mSalud de acuerdo a la clasificación propuesta por la Organización Mundial de la Salud ${ }^{(9)}$ en la Tabla 2. Los artículos se describen en la Tabla 3.
De los 19 artículos encontrados, 12 tuvieron como objetivo determinar el acceso, uso y percepciones de las tecnologías móviles entre los usuarios. El mayor número de esos estudios (8/12) estuvo relacionado a la 
Tabla 2. Categorías de mSalud, adaptado del informe de la Organización Mundial de la Salud (9)

\begin{tabular}{|c|c|}
\hline Campo de aplicación & Uso práctico \\
\hline \multicolumn{2}{|c|}{ Comunicación entre las personas y los servicios de salud } \\
\hline $\begin{array}{l}\text { Línea de ayuda telefónica en } \\
\text { salud }\end{array}$ & Asesoramiento vía telefónica sobre enfermedades \\
\hline $\begin{array}{l}\text { Llamadas telefónicas gratuitas } \\
\text { en caso de emergencia }\end{array}$ & Servicio para recibir información durante una emergencia \\
\hline \multicolumn{2}{|c|}{ Comunicación entre los servicios de salud y las personas } \\
\hline Adherencia al tratamiento & Envío de mensajes de voz o texto a los pacientes para el cumplimiento del tratamiento \\
\hline Recordatorios de citas & Envío de mensajes de voz o texto a los pacientes para programar o asistir a una cita \\
\hline $\begin{array}{l}\text { Promoción y concientización } \\
\text { sobre temas de salud }\end{array}$ & $\begin{array}{l}\text { Envío de mensajes de texto para sensibilizar a la población sobre temas de salud y/o para } \\
\text { comunicar sobre campañas de salud }\end{array}$ \\
\hline \multicolumn{2}{|c|}{ Consultas entre profesionales de la salud } \\
\hline Telemedicina & $\begin{array}{l}\text { Comunicación entre profesionales de la salud en zonas urbanas y rurales a través de un } \\
\text { dispositivo móvil (mensajes de texto, datos, imágenes o vídeo) para entrenamiento entre pa- } \\
\text { res, resolver dudas diagnósticas, mejorar la calidad de la atención y reducir las referencias } \\
\text { innecesarias }\end{array}$ \\
\hline \multicolumn{2}{|c|}{ Comunicación intersectorial en emergencias } \\
\hline Emergencias & $\begin{array}{l}\text { Uso de dispositivos móviles para controlar las situaciones de emergencia, como desastres } \\
\text { naturales o brotes de enfermedades. }\end{array}$ \\
\hline \multicolumn{2}{|c|}{ Monitoreo de enfermedades y vigilancia epidemiológica } \\
\hline $\begin{array}{l}\text { Recolección de información y } \\
\text { vigilancia epidemiológica }\end{array}$ & $\begin{array}{l}\text { Uso de dispositivos móviles para la recopilación de información y transmitir datos que se utili- } \\
\text { zarán para la vigilancia de enfermedades }\end{array}$ \\
\hline Seguimiento de pacientes & Uso de las tecnologías móviles para supervisar a los pacientes desde un lugar remoto \\
\hline \multicolumn{2}{|c|}{ Acceso a la información desde el lugar de atención } \\
\hline $\begin{array}{l}\text { Sistemas de apoyo a la toma de } \\
\text { decisiones }\end{array}$ & $\begin{array}{l}\text { Servicios que proporcionan acceso a las publicaciones científicas, bases de datos o herra- } \\
\text { mientas de diagnóstico en el lugar de atención }\end{array}$ \\
\hline Registros clínicos & El uso de dispositivos móviles para el ingreso y visualización de los registros de pacientes \\
\hline
\end{tabular}

aceptabilidad de los dispositivos electrónicos móviles como parte de un sistema dentro del manejo de la salud sexual y reproductiva (SSR); dos se basaron en diabetes mellitus (DM), y los otros dos en tuberculosis (TBC) e hipertensión (HTA). El resto de artículos (7/19) fueron estudios de intervención: cuatro en TBC, dos en SSR, y uno en dengue. Cinco (5/7) de estos estuvieron relacionados con el registro de información y vigilancia epidemiológica; uno trató sobre la promoción y concientización de temas de salud, y el último sobre telemedicina. De acuerdo a los dispositivos electrónicos móviles utilizados, 10/19 usaron los celulares (seis de estos utilizaron SMS), 6/19 los PDA y tres estaban relacionados a las TIC en general.

\section{ACCESO, USO Y PERCEPCIONES}

Se registran estudios publicados desde el 2007 en Perú, encontrando que los celulares y PDA podrían ser útiles y culturalmente relevantes para ayudar en la mejora de la adherencia en el tratamiento de diversas enfermedades. Esta categoría incluye a doce estudios que se realizaron en zonas urbanas, periurbanas de Lima y Callao. Además, siete estudios fueron cualitativos, cuatro cuantitativos y uno fue un estudio de caso. La mayoría de estudios (8/12) están relacionados a la SSR, cuya población objetivo fueron pacientes viviendo con VIH/SIDA (PVVS), hombres que tienen sexo con hombres $(\mathrm{HSH})$ y mujeres transgénero (MTG).

En la revisión se encontró que los PVVS se muestran muy positivos hacia la utilización de estos dispositivos como medios de apoyo y/o recordatorio para el tratamiento antirretroviral (11-14); además, el uso de estas tecnologías en HSH y MTG con VIH fomentarían cambios de comportamiento y reducción de conductas de riesgo ${ }^{(21)}$. Dos estudios evaluaron el uso de los SMS en HSH con la finalidad de encontrar el mensaje más apropiado para promover el uso de pruebas de detección de VIH en dicha población ${ }^{(18,19)}$, señalando que los mensajes deben ser cortos, neutros y no repetitivos ${ }^{(19)}$. Esta aceptación de los usuarios peruanos 
Tabla 3. Características generales de los artículos sobre la utilización de mSalud en Perú

\begin{tabular}{|c|c|c|c|c|c|}
\hline & Tema & MEDs & Población & Objetivo & Conclusión \\
\hline \multicolumn{6}{|c|}{ Acceso, uso y percepciones } \\
\hline $\begin{array}{l}\text { Curioso WH, } \\
2007\left({ }^{11}\right)\end{array}$ & $\begin{array}{l}\text { Salud sexual y } \\
\text { reproductiva }\end{array}$ & TIC & 31 PVS en Lima & $\begin{array}{l}\text { Evaluar la percepción que tienen las PVS respecto } \\
\text { a la tecnología como medio de apoyo para el } \\
\text { tratamiento antirretroviral. }\end{array}$ & $\begin{array}{l}\text { Las PVVS se muestran positivas frente a la utilización del } \\
\text { Internet, celulares y PDA como medios para promover su } \\
\text { salud. }\end{array}$ \\
\hline $\begin{array}{l}\text { Kurth AE, } \\
2007\left({ }^{12}\right)\end{array}$ & $\begin{array}{l}\text { Salud sexual y } \\
\text { reproductiva }\end{array}$ & PDA & 31 PVS en Lima & $\begin{array}{l}\text { Examinar las percepciones de las PVS en torno a } \\
\text { la utilización de PDA como medio de apoyo para la } \\
\text { adherencia al tratamiento antirretroviral. }\end{array}$ & $\begin{array}{l}\text { El uso de las PDA puede ser una manera culturalmente } \\
\text { apropiada de ayuda a la adherencia en el tratamiento } \\
\text { antirretroviral. }\end{array}$ \\
\hline $\begin{array}{l}\text { Curioso WH, } \\
2008\left({ }^{13}\right)\end{array}$ & $\begin{array}{l}\text { Salud sexual y } \\
\text { reproductiva }\end{array}$ & PDA & 15 PVS en Lima & $\begin{array}{l}\text { Determinar el nivel de aceptación en la utilización de } \\
\text { PDA como soporte al tratamiento antirretroviral. }\end{array}$ & $\begin{array}{l}\text { Los pacientes calificaron las PDA como una herramienta } \\
\text { innovadora, fácil de usar, confiable y útil para el tratamiento } \\
\text { antirretroviral. }\end{array}$ \\
\hline $\begin{array}{l}\text { Curioso WH, } \\
2009\left({ }^{14}\right)\end{array}$ & $\begin{array}{l}\text { Salud sexual y } \\
\text { reproductiva }\end{array}$ & $\begin{array}{l}\text { Teléfonos } \\
\text { celulares } \\
\text { (SMS) }\end{array}$ & 26 PVV en Lima & $\begin{array}{l}\text { Caracterizar estrategias efectivas de recordatorios } \\
\text { en el tratamiento retroviral para PVVS a través de } \\
\text { los SMS. }\end{array}$ & $\begin{array}{l}\text { Los pacientes se mostraron positivos frente a la utilización } \\
\text { de SMS como recordatorios para el tratamiento del VIH. Los } \\
\text { pacientes mencionaron que les gustarian que los SMS fueran } \\
\text { cortos, sencillos y que utilizaran un lenguaje codificado. }\end{array}$ \\
\hline $\begin{array}{l}\text { Curioso WH, } \\
2009\left({ }^{15}\right)\end{array}$ & $\begin{array}{l}\text { Diabetes } \\
\text { mellitus }\end{array}$ & $\mathrm{TIC}$ & $\begin{array}{l}200 \text { pacientes con } \\
\text { DM }\end{array}$ & $\begin{array}{l}\text { Reportar el uso y percepciones hacia las TIC de } \\
\text { pacientes con DM. }\end{array}$ & $\begin{array}{l}\text { A pesar de que el uso de las TIC es relativamente bajo, la } \\
\text { percepción hacia un sistema o programa usando celulares o } \\
\text { Internet para el soporte de pacientes con DM es positiva. }\end{array}$ \\
\hline $\begin{array}{l}\text { Zolfo M, } \\
2010\left({ }^{16}\right)^{*}\end{array}$ & $\begin{array}{l}\text { Salud sexual y } \\
\text { reproductiva }\end{array}$ & $\begin{array}{l}\text { Teléfonos } \\
\text { celulares }\end{array}$ & $\begin{array}{l}20 \text { trabajadores de } \\
\text { salud }\end{array}$ & $\begin{array}{l}\text { Evaluar el acceso y el uso de módulos virtuales en } \\
\text { los celulares para reforzar el conocimiento clínico } \\
\text { sobre VIH en los profesionales de la salud. }\end{array}$ & $\begin{array}{l}\text { El uso de celulares puede ser una opción de aprendizaje } \\
\text { viable para el personal de salud en entornos con recursos } \\
\text { limitados. Sin embargo, el alto costo de los teléfonos } \\
\text { inteligentes puede ser una desventaja. }\end{array}$ \\
\hline $\begin{array}{l}\text { Curioso WH, } \\
2011\left({ }^{17}\right)\end{array}$ & $\begin{array}{l}\text { Diabetes } \\
\text { mellitus }\end{array}$ & $\mathrm{TIC}$ & $\begin{array}{l}7 \text { médicos } \\
\text { endocrinólogos en } \\
\text { Lima }\end{array}$ & $\begin{array}{l}\text { Describir el acceso y uso de las TIC de parte } \\
\text { de médicos endocrinólogos y describir sus } \\
\text { percepciones respecto a un sistema informático para } \\
\text { mejorar la adherencia al tratamiento en pacientes } \\
\text { con diabetes. }\end{array}$ & $\begin{array}{l}\text { La mayoría de los médicos endocrinólogos reportaron } \\
\text { aceptación hacia el uso de las TIC en su práctica clínica } \\
\text { y hacia el sistema en particular, refiriendo que es fácil de } \\
\text { utilizar. }\end{array}$ \\
\hline $\begin{array}{l}\text { Blas MM, } \\
2013\left({ }^{18}\right)\end{array}$ & $\begin{array}{l}\text { Salud sexual y } \\
\text { reproductiva }\end{array}$ & $\begin{array}{l}\text { Teléfonos } \\
\text { celulares } \\
\text { (SMS) }\end{array}$ & $60 \mathrm{HSH}$ en Lima & $\begin{array}{l}\text { Determinar el tipo de mensaje virtual más adecuado } \\
\text { para promover que los HSH se realicen pruebas de } \\
\text { detección de VIH. }\end{array}$ & $\begin{array}{l}\text { Los mensajes no deben ser estigmatizantes. A través de } \\
\text { estos, se debe concientizar a los HSH sobre las conductas } \\
\text { de riesgo y ayudar a reducir los temores de realizarse las } \\
\text { pruebas de detección del VIH. }\end{array}$ \\
\hline $\begin{array}{l}\text { Menacho } \\
\text { LA, } \\
2013\left({ }^{19}\right)\end{array}$ & $\begin{array}{l}\text { Salud sexual y } \\
\text { reproductiva }\end{array}$ & $\begin{array}{l}\text { Teléfonos } \\
\text { celulares } \\
\text { (SMS) }\end{array}$ & $62 \mathrm{HSH}$ en Lima & $\begin{array}{l}\text { Identificar las características que los SMS deben } \\
\text { tener a fin de motivar el tamizaje de VIH en HSH. }\end{array}$ & $\begin{array}{l}\text { Los SMS pueden deben ser cortos, de lenguaje neutro y no } \\
\text { repetitivos para promover el tamizaje de VIH entre los HSH } \\
\text { peruanos. }\end{array}$ \\
\hline $\begin{array}{l}\text { Albino S, } \\
2014\left({ }^{20}\right)\end{array}$ & Tuberculosis & $\begin{array}{l}\text { Teléfonos } \\
\text { celulares } \\
\text { (SMS) }\end{array}$ & $\begin{array}{l}16 \text { pacientes con } \\
\text { TBC en Lima }\end{array}$ & $\begin{array}{l}\text { Examinar las percepciones y actitudes respecto a la } \\
\text { aceptabilidad de la utilización SMS para aumentar } \\
\text { adherencia al tratamiento de pacientes con TBC. }\end{array}$ & $\begin{array}{l}\text { Esta tecnología puede ser un medio eficaz para enviar } \\
\text { textos motivacionales sobre el tratamiento, información en } \\
\text { salud y recordatorios sencillos para aumentar la adherencia } \\
\text { al tratamiento de los pacientes con TBC. }\end{array}$ \\
\hline $\begin{array}{l}\text { Krishnan A, } \\
2014\left({ }^{21}\right)\end{array}$ & $\begin{array}{l}\text { Salud sexual y } \\
\text { reproductiva }\end{array}$ & $\begin{array}{l}\text { Teléfonos } \\
\text { celulares }\end{array}$ & $\begin{array}{l}339 \text { personas, entre } \\
\text { HSH y MTG }\end{array}$ & $\begin{array}{l}\text { Examinar el acceso, uso y aceptabilidad de } \\
\text { la utilización celulares en HSH y MTG para la } \\
\text { prevención de ITS. }\end{array}$ & $\begin{array}{l}\text { Las estrategias de intervención basadas en el uso de } \\
\text { teléfonos celulares (llamadas y SMS) son aceptadas por los } \\
\text { HSH y MTG para evaluar y reducir sus comportamientos } \\
\text { de riesgo. }\end{array}$ \\
\hline $\begin{array}{l}\text { Diez- } \\
\text { Canseco F, } \\
2015\left({ }^{22}\right)\end{array}$ & Hipertensión & $\begin{array}{l}\text { Teléfonos } \\
\text { celulares } \\
\text { (SMS) }\end{array}$ & $\begin{array}{l}108 \text { participantes } \\
\text { ( } 36 \text { de Perú) }\end{array}$ & $\begin{array}{l}\text { Validar un conjunto de SMS que serán utilizados } \\
\text { para promover cambios de comportamiento hacia la } \\
\text { prevención de la HTA. }\end{array}$ & $\begin{array}{l}\text { Los SMS tuvieron tasas muy altas de comprensión }(97 \%) \text { y } \\
\text { fueron considerados atractivos (puntaje promedio de } 8.7 / 10 \text { ) } \\
\text { por el total de participantes. }\end{array}$ \\
\hline \multicolumn{6}{|c|}{ Registro de información y vigilancia epidemiológica } \\
\hline $\begin{array}{l}\text { Curioso WH, } \\
2005\left({ }^{23}\right)\end{array}$ & $\begin{array}{l}\text { Salud sexual y } \\
\text { reproductiva }\end{array}$ & $\begin{array}{l}\text { Teléfonos } \\
\text { celulares }\end{array}$ & $\begin{array}{l}\text { Seis agentes } \\
\text { comunitarios de Ica, } \\
\text { Huánuco y Piura } \\
\text { que enviaron } 797 \\
\text { reportes }\end{array}$ & $\begin{array}{l}\text { Desarrollar un sistema usando teléfonos celulares } \\
\text { para la recolección y transmisión de eventos } \\
\text { adversos relacionados a la administración de } \\
\text { fármacos. }\end{array}$ & $\begin{array}{l}\text { Los teléfonos celulares son un recurso viable de recolección } \\
\text { y reporte de datos en tiempo real sobre los efectos adversos } \\
\text { de los fármacos (metronidazol). }\end{array}$ \\
\hline $\begin{array}{l}\text { Blaya JA, } \\
2006\left({ }^{24}\right)\end{array}$ & Tuberculosis & PDA & $\begin{array}{l}\text { Centros de atención } \\
\text { primaria en salud en } \\
\text { Lima y en el Callao }\end{array}$ & $\begin{array}{l}\text { Comparar el sistema de recolección bacteriológica } \\
\text { de TBC utilizando PDA frente al sistema basado } \\
\text { en papel. }\end{array}$ & $\begin{array}{l}\text { El sistema basado en PDA puede disminuir } \\
\text { significativamente el tiempo invertido en el proceso de } \\
\text { recolección de datos así como los errores durante el } \\
\text { procesamiento de la información. }\end{array}$ \\
\hline $\begin{array}{l}\text { Bernabe- } \\
\text { OrtizA, } \\
2008\left({ }^{25}\right)\end{array}$ & $\begin{array}{l}\text { Salud sexual y } \\
\text { reproductiva }\end{array}$ & PDA & $\begin{array}{l}398 \text { participantes } \\
\text { de Lima }\end{array}$ & $\begin{array}{l}\text { Evaluar la calidad de la recolección datos sensibles } \\
\text { sobre sexualidad usando PDA, y compararlo al } \\
\text { sistema basado en papel. }\end{array}$ & $\begin{array}{l}\text { La utilización de un sistema basado en PDA es una } \\
\text { alternativa viable para la recolección de datos, puesto que } \\
\text { existe un alto nivel de concordancia con las respuestas } \\
\text { obtenidas en papel, además de reducir las incongruencias } \\
\text { y datos perdidos. }\end{array}$ \\
\hline $\begin{array}{l}\text { Blaya JA, } \\
2008\left({ }^{26}\right)\end{array}$ & Tuberculosis & PD & $\begin{array}{l}126 \text { laboratorios y } \\
\text { centros de atención } \\
\text { primaria en salud de } \\
\text { Lima y Callao. }\end{array}$ & $\begin{array}{l}\text { Evaluar la eficacia de la recolección de información } \\
\text { de pacientes con TBC a través de PDA frente al } \\
\text { sistema basado en papel. }\end{array}$ & $\begin{array}{l}\text { El sistema basado en PDA disminuyó drásticamente el } \\
\text { esfuerzo y tiempo para recolectar los resultados de TBC, } \\
\text { por lo que su aplicación puede ser beneficiosa en lugares de } \\
\text { bajos recursos. }\end{array}$ \\
\hline $\begin{array}{l}\text { Blaya JA, } \\
2009\left({ }^{27}\right)\end{array}$ & Tuberculosis & PDA & $\begin{array}{l}93 \text { centros de } \\
\text { atención primaria en } \\
\text { salud de Lima. }\end{array}$ & $\begin{array}{l}\text { Evaluar la eficacia de la utilización de PDA para } \\
\text { recolectar resultados de pruebas de TBC y } \\
\text { compararla al sistema basado en papel. }\end{array}$ & $\begin{array}{l}\text { El sistema basado en PDA redujo significativamente el } \\
\text { tiempo del procesamiento de los datos, la frecuencia de } \\
\text { errores al igual que el número de horas trabajadas. }\end{array}$ \\
\hline \multicolumn{6}{|c|}{ Promoción y concientización sobre temas de salud } \\
\hline $\begin{array}{l}\text { Dammert } \\
\text { AC, } \\
2014\left({ }^{28}\right)\end{array}$ & Dengue & $\begin{array}{l}\text { Teléfonos } \\
\text { celulares } \\
\text { (SMS) }\end{array}$ & $\begin{array}{l}2021 \text { dueños } \\
\text { de casa en } 100 \\
\text { localidades en Piura }\end{array}$ & $\begin{array}{l}\text { Evaluar la eficacia del envío de información a través } \\
\text { de celulares para promover conductas preventivas } \\
\text { contra el dengue. }\end{array}$ & $\begin{array}{l}\text { El envío de SMS sugiere que la exposición repetida a } \\
\text { la información genera cambios de comportamientos } \\
\text { relacionados a la prevención del dengue. }\end{array}$ \\
\hline \multicolumn{6}{|l|}{ Telemedicina } \\
\hline $\begin{array}{l}\text { Zimic M, } \\
2009\left({ }^{29}\right)\end{array}$ & Tuberculosis & $\begin{array}{l}\text { Teléfonos } \\
\text { celulares }\end{array}$ & $\begin{array}{l}75 \text { imágenes } \\
\text { transmitidas }\end{array}$ & $\begin{array}{l}\text { Evaluar la utilidad de los celulares como parte de } \\
\text { un sistema integrado para facilitar el diagnóstico de } \\
\text { TBC a distancia. }\end{array}$ & $\begin{array}{l}\text { La concordancia entre la observación directa en el } \\
\text { microscopio y la imagen transmitida por el celular fue casi } \\
\text { perfecta, por lo que su uso facilita el diagnóstico de TBC en } \\
\text { zonas remotas. }\end{array}$ \\
\hline
\end{tabular}

TIC: tecnologías de la información y la comunicación; PDA: personal digital assistants; SMS: mensajes de texto; PVVS: personas viviendo con VIH/SIDA; HSH: hombres que tienen sexo con hombres; MTG: mujeres transgénero; DM: diabetes mellitus; TBC: tuberculosis; HTA: hipertensión arterial; ITS: Infecciones de transmisión sexual.

*Artículo también considerado en la categoría "Telemedicina". 
hacia la utilización de la mSalud es de vital importancia, puesto que se ha demostrado que los mensajes de texto aumentan la adherencia al tratamiento en PVS en regiones donde los recursos son escasos y las poblaciones están muy alejadas ${ }^{(30)}$. Por último, un estudio evaluó el acceso y uso de los teléfonos inteligentes para la educación en VIH a profesionales de la salud, encontrando que podría ser una opción en lugares alejados a pesar de su costo ${ }^{(16)}$. En general, estos resultados crean una base para futuras intervenciones y estudios más complejos que evalúen efectividad y uso de esta tecnología.

Por otro lado, a pesar de su alto impacto en salud pública, solo se encontró un estudio relacionado a la TBC (20). Este estudio evaluó la posibilidad del uso de SMS para aumentar la adherencia del tratamiento antituberculoso. Esto ya ha sido demostrado anteriormente (31) y los resultados han sido replicables en Perú (20), por lo que existiría un escenario adecuado para la implementación de esta tecnología en nuestro país.

De la misma manera, dos estudios evaluaron el uso de las TIC en enfermedades crónicas como DM, encontrando una aceptación adecuada ${ }^{(15,17)}$. Otro estudio validó el uso de SMS para la prevención primaria (promoción de estilos de vida saludable) de la HTA en LA en donde el Perú fue uno de los tres países incluidos ${ }^{(22)}$. Los resultados fueron positivos y alentadores, con altas tasas de comprensión y atractividad hacia el usuario ${ }^{(22)}$, por lo que la propuesta presenta gran potencial en el uso de mSalud para mejorar las conductas relacionadas con el estilo de vida para la prevención de la HTA y otras enfermedades crónicas no transmisibles.

\section{REGISTRO DE INFORMACIÓN Y VIGILANCIA EPIDEMIOLÓGICA}

Si bien se registran trabajos desde el año 2005, y principalmente utilizando PDA, los cinco estudios muestran resultados positivos. Tres estudios se enfocaron en la TBC (24-27), una enfermedad vigente en nuestro entorno. En esos trabajos se encontró que el uso de las tecnologías móviles redujo el tiempo de trabajo para recolectar la información bacteriológica, además de reducir las incongruencias y errores asociados al reporte basado en papel. Los otros dos trabajos estuvieron relacionados a la SSR. En el primero, los celulares fueron una fuente viable de registro y reporte de datos en tiempo real sobre los efectos adversos de los fármacos (metronidazol) (23); mientras que en el otro, las PDA demostraron ser una alternativa para la recolección de datos sensibles sobre la sexualidad, con una alta concordancia respecto a las respuestas obtenidas en papel ${ }^{(25)}$.
Los análisis de costos muestran que estos sistemas son capaces de recuperar los altos costos de inversión iniciales, proporcionando una mayor eficiencia y reducción de costos a largo plazo ${ }^{(6)}$. Blaya et al. describen que en el análisis de ocho estudios realizados en África ${ }^{(6)}$, los gastos después de usar el sistema basado en PDA serían igual a los del sistema en papel para la recolección de información. De la misma manera, el análisis en Uganda mostró un ahorro de $91 \%$ sobre el sistema de papel (6) y que el sistema de PDA en el Perú podría ser costoefectivo si se llegase a expandir ${ }^{(6)}$. Resultados similares se han encontrado al utilizar celulares en otros entornos. Por ejemplo, en México se llevó a cabo un estudio con celulares para recopilar datos sobre el control vectorial

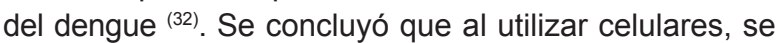
eliminó la necesidad de la entrada manual de datos a un formulario en papel, evitando los errores de transcripción de datos y agilizando el proceso de transmisión de los datos a una base central para la toma de decisiones respecto a las estrategias del control vectorial del dengue ${ }^{(32)}$.

En general, la utilización de la mSalud en la recolección de datos y vigilancia epidemiológica es eficiente y una alternativa a considerar. Estos trabajos son la base que permite justificar proyectos con mayor complejidad, basados en mSalud, para la mejoría de estos procesos.

\section{PROMOCIÓN SOBRE TEMAS DE SALUD}

Se encontró solo un artículo publicado en el Perú. Dammert et al. evaluaron la eficacia de enviar información a través de celulares (SMS) para promover conductas hacia la prevención del dengue ${ }^{(28)}$. Este trabajo se llevó a cabo en una localidad de Piura, y a través de la intervención aleatorizada de 3 meses de duración, se demostró que el indicador (cantidad de recipientes con presencia de larvas) fue menor en las casas en donde se enviaron los mensajes de texto. Además, este proyecto también menciona ser costo-efectivo, debido a que con solo el envío de los SMS se estaría evitando el costo de tratamiento para el dengue. Esto no resulta sorpresivo puesto que se sabe que el envío de SMS promueve mejoras en la conducta de los pacientes, sea para mejorar la adherencia al tratamiento antituberculoso (31), antirretroviral (33) o estilos de vida saludables en pacientes con diabetes ${ }^{(34)}$ a un costo mínimo. Se espera que otros proyectos tomen una iniciativa similar y demuestren que la salud móvil puede ser una estrategia costo-efectiva a considerar para apoyar conductas preventivas y de manejo en enfermedades infecciosas y crónicas. 


\section{TELEMEDICINA}

El trabajo realizado por Zimic et al. (2009) se relacionó con la TBC y el uso de celulares para el diagnóstico a distancia ${ }^{299}$. El proyecto piloto utilizó el celular como medio de transmisión de imágenes provenientes de un microscopio para poder almacenarlas en la web para luego ser revisadas por un experto. Este trabajo demuestra que las imágenes de $130 \mathrm{~KB}$, en promedio, permiten una lectura adecuada del Microscopic Observation Drug Susceptibility (MODS) lo cual aborda un problema central de nuestro país: la centralización. Al utilizar los celulares como parte de un sistema integrado al MODS, el diagnóstico de TBC se podrá realizar a distancia en muchos centros del país de manera precoz y con una inversión mínima. De manera similar, Breslauer et al. demostraron que la microscopía de luz acoplada a un teléfono celular, proporciona un método sencillo, rentable, y vital para el diagnóstico por imágenes de glóbulos rojos infectados por $P$. falciparum y muestras de esputo infectadas por M. tuberculosis ${ }^{(35)}$.

Asimismo, es necesario recalcar que el trabajo realizado por Zolfo et al. (2010), descrito en la sección de "acceso, uso y percepciones", también evaluó el uso de los teléfonos inteligentes para la educación en VIH a profesionales de la salud, encontrando que los módulos educativos en los teléfonos móviles pueden dar flexibilidad a los trabajadores de salud para acceder a contenidos de aprendizaje en cualquier lugar. Sin embargo, la falta de interoperabilidad y el alto costo de la inversión para la compra de los teléfonos podrían representar una limitación para el amplio uso de este tipo de programas de teleducación ${ }^{(16)}$.

Este potencial para el diagnóstico de enfermedades en zonas alejadas, donde las instalaciones de laboratorio son prácticamente inexistentes, pero con amplia infraestructura móvil, podría ser pieza clave como estrategia de intervención en salud pública al aumentar la cantidad de personas diagnosticadas y tratadas. Asimismo, la teleeducación con tecnologías móviles representan un gran potencial para entregar información y diseñar programas educativos a personal de salud y usuarios de salud.

\section{CONCLUSIONES Y LECCIONES APRENDIDAS}

La literatura peruana revisada muestra que las intervenciones de mSalud, que van desde la entrega de información de salud usando celulares, al registro de datos a distancia y diagnóstico remoto, tienen un enorme potencial para mejorar el acceso y calidad de los servicios de salud, aumentando la efectividad de los programas de salud pública y reduciendo los costos de la asistencia sanitaria ${ }^{(1,8)}$. De la misma manera, los estudios realizados en nuestro país han demostrado que las tecnologías móviles son, en general, aceptadas por la población y que su uso apropiado en el sector salud contribuiría a disminuir las brechas en la asistencia médica, reduciendo las limitaciones tales como la falta de recursos (profesionales de salud y equipamiento en los establecimientos de salud), la alta dispersión de la población y la escasez de infraestructura (carreteras, transporte, y conectividad) (7). Asimismo, resulta necesario evaluar el impacto de aplicaciones diseñadas para los teléfonos inteligentes, tablets u otros dispositivos móviles (app) como soporte para los profesionales de salud y para los pacientes y usuarios de servicios de salud.

La mayoría de los estudios incluidos en este trabajo tuvieron como escenario Lima o Callao, por lo que al no ser estudios a escala nacional, no son necesariamente representativos de la realidad peruana y su aplicabilidad en poblaciones más vulnerables y alejadas merece evaluación en futuros estudios. De la misma manera, solo 5/19 estudios trataron sobre la TBC a pesar de representar un problema para la salud pública en el Perú ${ }^{(36)}$, y $3 / 19$ se relacionaron con enfermedades crónicas, pese a las consecuencias de la transición epidemiológica y el aumento de la prevalencia de dichas enfermedades en Perú ${ }^{\left({ }^{37}\right)}$. Una de las desventajas de los estudios de esta sección es que 6 de ellos evaluaron el uso de PDA que actualmente se encuentra en desuso, pero que podrían ser replicadas en teléfonos inteligentes (smartphones).

Otra limitación encontrada es que el cuerpo de la literatura peruana de mSalud se basa en proyectos pilotos sobre la viabilidad, ejecución, adopción, el uso y la aceptación de tecnologías implementadas por un período corto; este hallazgo es similar a lo reportado por Chigona et al. en su revisión de la aplicación de mSalud en países en vías de desarrollo ${ }^{(38)}$. Es importante contar con estudios sobre la eficacia, costo-efectividad y el impacto de estas intervenciones a escala nacional. Para ello, es necesario que más instituciones contribuyan con generar evidencia necesaria para que los gobiernos lo incorporen como políticas públicas y no queden solo como proyectos pilotos ${ }^{(38,39)}$. Por ese motivo, se deben desarrollar estrategias innovadoras que permitan superar algunos desafíos pendientes como lo son: 1) la falta de conocimiento y sensibilización de los responsables de las decisiones de los países acerca de las ventajas y beneficios de la tecnología móvil para los sistemas de atención de la salud; 2 ) la falta de formación de los profesionales de la salud y falta de programas de 
entrenamiento en informática biomédica, telemedicina y salud móvil; 3) el débil liderazgo y coordinación de proyectos incluyendo estrategias de gestión del cambio; 4) falta de incentivos a profesionales de salud para promover el uso eficiente a estas tecnologías; y 5) alianzas insuficientes entre los proveedores de servicios e instituciones, incluyendo alianzas público-privadas ${ }^{(8)}$.

Recientemente el Consejo Nacional de Ciencia, Tecnología e Innovación Tecnológica (CONCYTEC) en alianza con la agencia Grand Challenges Canadá ha financiado proyectos innovadores relacionados a salud móvil (40). Sin embargo, se requiere de mayor investigación en estas áreas para comprender en su totalidad el potencial de la mSalud para que puedan ser base y sustento de políticas de estado innovadoras, integradas al sistema de salud y costo-efectivas para la mejora en la atención e inclusión en salud en el país ${ }^{(2,10)}$. Por esta razón, es necesaria la intervención del gobierno e instituciones de salud a invertir mayores recursos que permitan fomentar la investigación, publicación y difusión de estos tipos de proyectos en nuestro país.

Contribuciones de autoría: concepción y diseño del trabajo: EFR, AP, OJP, WHC; redacción del manuscrito: EFR, AP, OJP; revisión crítica del manuscrito: WHC; aprobación de su versión final: EFR, AP, OJP, WHC.

\section{Fuentes de financiamiento: autofinanciado}

Conflictos de interés: los autores declaran no tener conflictos de interés en la publicación del artículo

\section{REFERENCIAS BIBLIOGRÁFICAS}

1. Vital Wave Consulting. mHealth for Development: The Opportunity of Mobile Technology for Healthcare in the Developing World [Internet]. Washington, D.C.: UN FoundationVodafone Foundation Partnership; 2009 [citado el 16 de marzo de 2015]. Disponible en: http://goo.gl/GClZyq

2. Curioso WH, Mechael PN. Enhancing 'M-health' with south-to-south collaborations. Health Aff (Millwood). 2010;29(2):264-7. doi: 10.1377/ hlthaff.2009.1057.

3. International Telecommunication Union. The world in 2014: ICT facts and figures [Internet]. Geneva: ITU; 2014 [citado el 16 de marzo de 2015]. Disponible en: http://goo.gl/ MQ3w2r

4. Free C, Phillips G, Felix L, Galli L, Patel V, Edwards P. The effectiveness of M-health technologies for improving health and health services: a systematic review protocol. BMC Res Notes. 2010;3:250. doi: 10.1186/1756-0500-3-250.

5. World Economic Forum. Amplifying the impact: examining the intersection of mobile health and mobile finance [Internet]. Geneva: WEF; 2011 [citado el 16 de marzo de 2015]. Disponible en: http://goo.gl/qcF82d

6. Blaya JA, Fraser HS, Holt B. E-health technologies show promise in developing countries. Health Aff (Millwood). 2010;29(2):244-51. doi: 10.1377/hlthaff.2009.0894.

7. Prieto-Egido I, Simó-Reigadas J, LiñánBenítez L, García-Giganto V, Martínez-
Fernández A. Telemedicine Networks of EHAS Foundation in Latin America. Front Public Health. 2014; 2:188. doi: 10.3389/fpubh.2014.00188.

8. Aylward DK, Leão B, Curioso WH, Cruz F. Can you heal me now? Potential (and pitfalls) of mHealth. Americas Quarterly 2010;4(3):88-95.

9. World Health Organization. mHealth: New horizons for health through mobile technologies [Internet]. Geneva: WHO; 2011 [citado el 16 de marzo de 2015]. Disponible en: http:// goo.gl/pmIGBb

10. Curioso WH. Capítulo XII: Salud móvil en atención primaria. En: Carnicero J, Fernández A, Rojas D. Manual de Salud Electrónica para Directivos de Servicios y Sistemas de Salud. Vol II. Aplicaciones de las TIC a la atención primaria de salud [Internet]. Madrid: Sociedad Española de Informática de la Salud, Comisión Económica para América Latina y el Caribe y Naciones Unidas. España; 2014 [citado el 16 de marzo de 2015]. Disponible en: http://goo.gl/zkcBIY

11. Curioso WH, Kurth AE. Access, use and perceptions regarding Internet, cell phones and PDAs as a means for health promotion for people living with HIV in Peru. BMC Med Inform Decis Mak. 2007;7:24.

12. Kurth AE, Curioso WH, Ngugi E, McClelland L, Segura P, Cabello R, et al. Personal digital assistants for HIV treatment adherence, safer sex behavior support, and provider training in resource-constrained settings. AMIA Annu Symp Proc. 2007:1018.

13. Curioso WH, Kurth AE, Cabello R, Segura P, Berry DL. Usability evaluation of Personal Digital Assistants (PDAs) to support HIV treatment adherence and safer sex behavior in Peru. AMIA Annu Symp Proc. 2008:918.

14. Curioso WH, Quistberg DA, Cabello R, Gozzer E, Garcia PJ, Holmes KK, et al. "It's time for your life": How should we remind patients to take medicines using short text messages?. AMIA Annu Symp Proc. 2009;2009:129-33.

15. Curioso WH, Gozzer E, Valderrama M, Rodriguez-Abad J, Villena JE, Villena AE. Uso y percepciones hacia las tecnologías de información y comunicación en pacientes con diabetes, en un hospital público del Perú. Rev Peru Med Exp Salud Publica. 2009;26(2):161-67.

16. Zolfo M, Iglesias D, Kiyan C, Echevarria J, Fucay L, Llacsahuanga E, et al. Mobile learning for HIV/AIDS healthcare worker training in resourcelimited settings. AIDS Res Ther. 2010;7:35.

17. Curioso WH, Gozzer E, RodríguezAbad J. Acceso y uso de las tecnologías de información y comunicación y percepciones hacia un sistema para mejorar la adherencia al tratamiento, en médicos endocrinólogos de un hospital público del Perú. Rev Med Hered 2011;22(1):15-22.

18. Blas MM, Menacho LA, Alva IE, 
Cabello R, Orellana ER. Motivating men who have sex with men to get tested for HIV through the internet and mobile phones: a qualitative study. PLoS One. 2013;8(1):e54012. doi: 10.1371/journal.pone.0054012.

19. Menacho LA, Blas MM, Alva IE, Orellana RE. Short Text Messages to Motivate HIV Testing Among Men Who have Sex with Men: A Qualitative Study in Lima, Peru. Open AIDS J. 2013;7:1-6. doi: $10.2174 / 1874613601307010001$.

20. Albino S, Tabb KM, Requena D, Egoavil M, Pineros-Leano MF, Zunt JR, et al. Perceptions and acceptability of short message services technology to improve treatment adherence amongst tuberculosis patients in Peru: a Focus Group Study. PLoS One. 2014;9(5):e95770. doi: 10.1371/ journal.pone.0095770.

21. Krishnan A, Ferro EG, Weikum D, Vagenas P, Lama JR, Sanchez J, et al. Communication technology use and mHealth acceptance among HIV-infected men who have sex with men in Peru: implications for HIV prevention and treatment. AIDS Care. 2015;27(3):273-82. doi: 10.1080/09540121.2014.963014.

22. Diez-Canseco F, Zavala-Loayza JA, Beratarrechea A, Kanter R, RamirezZea M, Rubinstein A, et al. Design and Multi-Country Validation of Text Messages for an mHealth Intervention for Primary Prevention of Progression to Hypertension in Latin America. JMIR Mhealth Uhealth. 2015;3(1):e19. doi: 10.2196/mhealth.3874.

23. Curioso WH, Karras BT, Campos PE, Buendía C, Holmes KK, Kimball AM. Design and Implementation of CellPREVEN: A Real-Time Surveillance System for Adverse Events Using Cell Phones in Peru. AMIA Annu Symp Proc. 2005;2005:176-80.

24. Blaya J, Fraser HSF. Development, implementation and preliminary study of a PDA-based bacteriology collection system. AMIA Annu Symp Proc. 2006:2006:41-5.

25. Bernabe-OrtizA, Curioso WH, Gonzales MA, Evangelista W, Castagnetto JM, Carcamo CP, et al. Handheld computers for self-administered sensitive data collection: a comparative study in Peru. BMC Med Inform Decis Mak. 2008;8:11. doi: 10.1186/1472-69478-11.

26. Blaya JA, Gomez W, Rodriguez P, Fraser $\mathrm{H}$. Cost and implementation analysis of a personal digital assistant system for laboratory data collection. Int J Tuberc Lung Dis. 2008;12(8):921-7.

27. Blaya JA, Cohen T, Rodríguez P, Kim J, Fraser HSF. Personal digital assistants to collect tuberculosis bacteriology data in Peru reduce delays, errors, and workload, and are acceptable to users: cluster randomized controlled trial. Int J Infect Dis. 2009;13(3):410-8. doi: 10.1016/j.ijid.2008.09.015

28. Dammert AC, Galdo JC, Galdo V. Preventing dengue through mobile phones: evidence from a field experiment in Peru. J Health Econ. 2014;35:147-61.

29. Zimic M, Coronel J, Gilman RH, Luna CG, Curioso WH, Moore DA. Can the power of mobile phones be used to improve tuberculosis diagnosis in developing countries? Trans R Soc Trop Med Hyg. 2009;103(6):638-40. doi: 10.1016/j. trstmh.2008.10.015.

30. Pop-Eleches C, Thirumurthy $\mathrm{H}$, Habyarimana JP, Zivin JG, Goldstein MP, de Walque, et al. Mobile phone technologies improve adherence to antiretroviral treatment in a resource-limited setting: a randomized controlled trial of text message reminders. AIDS. 2011;25(6):825-34. doi: 10.1097/ QAD.0b013e32834380c1.

31. Nglazi MD, Bekker LG, Wood R, Hussey GD, Wiysonge CS. Mobile phone text messaging for promoting adherence to anti-tuberculosis treatment: a systematic review. BMC Infect Dis. 2013;13:566. doi: 10.1186/1471-2334-13-566.

32. Lozano-Fuentes S, Wedyan F, Hernandez-Garcia E, Sadhu D, Ghosh $\mathrm{S}$, Bieman JM, et al. Cell phonebased system (Chaak) for surveillance of immatures of dengue virus mosquito vectors. J Med Entomol. 2013;50(4):879-89.
33. Finitsis DJ, Pellowski JA, Johnson BT. Text message intervention designs to promote adherence to antiretroviral therapy (ART): a meta-analysis of randomized controlled trials. PLoS One. 2014;9(2):e88166. doi: 10.1371/ journal.pone.0088166.

34. Connelly J, Kirk A, Masthoff J, MacRury S. The use of technology to promote physical activity in Type 2 diabetes management: a systematic review. Diabet Med. 2013;30(12):1420-32. doi: 10.1111/ dme.12289.

35. Breslauer DN, Maamari RN, Switz NA, Lam WA, Fletcher DA. Mobile phone based clinical microscopy for global health applications. PLoS One. 2009;4(7):e6320. doi: 10.1371/ journal.pone.0006320.

36. Bonilla Asalde C. Situación de la tuberculosis en el Perú. Acta med. peruana. 2008;25(3):163-170.

37. Málaga G. Las enfermedades crónicas no transmisibles, un reto por enfrentar. Rev Peru Med Exp Salud Publica. 2014;31(1):6-8.

38. Chigona W, Nyemba-Mudenda M, Metfula AS. A review on mHealth research in developing countries. The Journal of Community Informatics [Internet]. 2013;9(2) [citado el $16 \mathrm{de}$ marzo de 2015]. Disponible en: http:// goo.gl/zo14SO

39. Chib A, van Velthoven $\mathrm{MH}$ Car J. mHealth adoption in lowresource environments: a review of the use of mobile healthcare in developing countries. J Health Commun. 2015;20(1):4-34. doi: 10.1080/10810730.2013.864735.

40. Consejo Nacional de Ciencia. Tecnología e Innovación Tecnológica. Ciencia Activa. Innovación para el Desarrollo. Proyectos Ganadores de Ideas Audaces. Lima; CONCYTEC [citado el 16 de marzo de 2015]. Disponible en: http://goo.gl/RgQYqh

Correspondencia: Eloy F. Ruiz Dirección: Av. El Polo 740 - Edificio C, oficina 313

Teléfono: 942023055

Correoelectrónico:eloy.ruiz.m@upch.pe 